\title{
Parameter Analysis and Experimental Investigations on Cylindrical Broadband Acoustic Holography
}

\author{
YANG DE-SEN \\ College of Underwater Acoustic Engineering, Harbin \\ Engineering University \\ Harbin 150001, China
}

HU BO

College of Underwater Acoustic Engineering, Harbin Engineering University

Harbin 150001, China

\begin{abstract}
Broadband acoustic holography based on intensity measurement (BAHIM) is a new technique for the reconstruction of sources. In BAHIM method, the acoustic intensity vector and potential energy density, which are both quadratic quantities, on the measurement surface are used to obtain the pressure phase. The principle of the cylindrical BAHIM theory is presented. In addition, the numerical simulations have been conducted. The influences of the change of parameters are discussed in detail. At last, the validity of BAHIM method and the correctness of parameter analysis are assessed by underwater near-field acoustic holography experiments. The experimental results show the merits of the underwater cylindrical BAHIM in the reconstruction of sound field, identification and localization of the noise sources.
\end{abstract}

Keywords- cylindrical BAHIM; parameter analysis; intensity measurement

\section{INTRODUCTION}

Near-field Acoustical Holography (NAH) [1] was a new technique coming from acoustical holography in the 1980s. Compared with conventional acoustical holography, it is possible in NAH to image more than just the acoustic pressure. One can obtain the three components of the fluid velocity and acoustic intensity from the surface of the acoustic source into the far field [2-4].

Broadband acoustic holography from intensity measurements (BAHIM) [5-6] was first introduced in 1987. In BAHIM method, the main advantage is the measurement practicability in comparison with the technique in which the pressure phase is measured directly without an array of referenced microphones or by use of the reference signal to the source signal. Therefore, it can be well used with broadband acoustic spectrum sources, and the technique is not limited to laboratory cases where source excitation is controlled.

\author{
SUN YU \\ College of Underwater Acoustic Engineering, Harbin \\ Engineering University \\ Harbin 150001, China
}

In this paper, we review the theory of cylindrical BAHIM and describe the validity of the BAHIM method in the cylindrical geometric source surface by computer, and the errors which arise from the changes of parameters. At last, the experimental results show the merits of the BAHIM in the reconstruction of sound field, the correctness of parameter analysis in simulations, and the feasibility of the using of vector hydrophone array in the NAH measurement.

\section{PRINCIPLE OF BAHIM THEORY}

With the principle of cylindrical near-field acoustic holography [2], the complex pressure Fourier transform $p_{\mathrm{H}}\left(r_{\mathrm{H}}, \theta, z\right)$ specified on the hologram cylinder is

$$
\begin{aligned}
p_{\mathrm{H}}\left(r_{\mathrm{H}}, m, k_{z}\right)= & \frac{1}{2 \pi} \int_{0}^{2 \pi} \int_{-\infty}^{+\infty} p_{\mathrm{H}}\left(r_{\mathrm{H}}, \theta, z\right) \\
& \times e^{j m \theta} e^{j k_{z} z} \mathrm{~d} \theta \mathrm{d} z
\end{aligned}
$$

where $m$ is the index of the circumferential wave numbers and $k_{z}$ is the axial wave numbers.

The Fourier transform of the pressure distribution on the cylindrical source of radius $r_{s}$ is expressed as

$$
p_{\mathrm{H}}\left(r_{\mathrm{H}}, m, k_{z}\right)=p_{\mathrm{S}}\left(r_{\mathrm{S}}, m, k_{z}\right) \times G_{m}\left(k_{z}, r_{\mathrm{H}} / r_{\mathrm{S}}\right)
$$

where $G_{m}\left(k_{z}, r_{\mathrm{H}} / r_{\mathrm{S}}\right)$ is the Green function.

$$
G_{m}\left(k_{z}, r_{\mathrm{H}} / r_{\mathrm{S}}\right)=H_{m}^{(1)}\left(k_{r} r_{\mathrm{H}}\right) / H_{m}^{(1)}\left(k_{r} r_{\mathrm{S}}\right)
$$

$\mathrm{NAH}$ requires the knowledge of amplitude and phase of the complex pressure on the cylindrical hologram. The complex pressure $p_{\mathrm{H}}$ is

$$
p_{\mathrm{H}}\left(r_{\mathrm{H}}, \theta, z\right)=P_{\mathrm{H}}\left(r_{\mathrm{H}}, \theta, z\right) e^{j \varphi_{\mathrm{H}}\left(r_{\mathrm{H}}, \theta, z\right)}
$$


In BAHIM method, $P_{\mathrm{H}}$ can be measured, the key is the relationship between active intensity and the spatial gradient of the complex pressure phase

$$
\begin{gathered}
I_{m}\left(r_{\mathrm{H}}, \theta, z, \omega\right)=\frac{1}{2 \omega \rho}\left|P_{\mathrm{H}}\left(r_{\mathrm{H}}, \theta, z, \omega\right)\right|^{2} \\
\cdot \nabla \varphi_{\mathrm{H}}\left(r_{\mathrm{H}}, \theta, z, \omega\right)
\end{gathered}
$$

where $\rho$ is the mass density in medium. If we only consider the components of the active intensity vector, equation (5) thus expresses the phase gradient on the cylindrical hologram as

$$
\begin{aligned}
& \frac{1}{r} \frac{\partial \varphi(\theta, z)}{\partial \theta} \theta+\frac{\partial \varphi(\theta, z)}{\partial z} z \\
= & -k\left(B_{\theta}(\theta, z) \theta+B_{z}(\theta, z) z\right)
\end{aligned}
$$

Taking the two-dimensional Fourier transform, we obtain for the phase

$$
\begin{gathered}
\varphi\left(m, k_{z}\right)=\frac{-j k\left(\frac{m}{r_{H}}\right)}{\left(\frac{m}{r_{H}}\right)^{2}+k_{z}^{2}} B_{\theta}\left(m, k_{z}\right) \\
+\frac{-j k k_{z}}{\left(\frac{m}{r_{H}}\right)^{2}+k_{z}^{2}} B_{z}\left(m, k_{z}\right) \\
b_{\theta}(\theta, z)=\frac{2 \rho c I_{\theta}(\theta, z)}{|p(\theta, z)|^{2}}, b_{z}(\theta, z)=\frac{2 \rho c I_{z}(\theta, z)}{|p(\theta, z)|^{2}}, \\
B_{\theta}\left(m, k_{z}\right)=\int_{-\infty}^{+\infty} \int_{-\infty}^{+\infty} b_{\theta}(\theta, z) \mathrm{e}^{-j\left(m \theta+k_{z} z\right)} \mathrm{d} \theta \mathrm{d} z, \\
B_{z}\left(m, k_{z}\right)=\int_{-\infty}^{+\infty} \int_{-\infty}^{+\infty} b_{z}(\theta, z) \mathrm{e}^{-j\left(m \theta+k_{z} z\right)} \mathrm{d} \theta \mathrm{d} z
\end{gathered}
$$

The complex pressure on the hologram is reconstructed by taking for the module the square root of the measured quadratic pressure and for the phase the inverse transform of the preceding expression

\section{PARAMETER ANALYSIS}

From the distribution of the holographic reconstructed phase error of the point source in [7], we can conclude that the periodic fluctuant error exists obviously in the process of the phase reconstruction. The effect of the reconstructed parameters on the reconstructed phase error and the reconstructed precision are emphasized here.

Several examples make it possible to evaluate the parameter analysis using computer simulations. In Fig. 1, the center of cylinder is the coordinate center, and $z$ is the axis of the cylinder. The acoustic field is assumed to be produced by a point source with the position of $(0.3,0,0)$ in cylindrical coordinate. The point source frequency is $1500 \mathrm{~Hz}$ on a finite holographic cylindrical surface with a gird of $40 \times 40$ points. The holographic cylinder radius $r_{\mathrm{H}}$ and reconstructed cylinder radius $r_{\mathrm{S}}$ are $0.4 \mathrm{~m}$ and $0.32 \mathrm{~m}$ respectively. The sound speed $c$ in water is $1500 \mathrm{~m} / \mathrm{s}$. The water density $\rho$ is $1000 \mathrm{~kg} / \mathrm{m}^{3}$.

In order to evaluate the difference of the theoretical and reconstructed pressure phase, the absolute phase error $\Delta \varphi\left(r_{\mathrm{H}}, \theta, z\right)$ the maximal phase error $\varepsilon_{\varphi \max }$ and the average phase error $\overline{\varepsilon_{\varphi}}$ are defined as

$$
\begin{aligned}
\Delta \varphi\left(r_{\mathrm{H}}, \theta, z\right) & =\varphi_{r e}\left(r_{\mathrm{H}}, \theta, z\right)-\varphi_{l}\left(r_{\mathrm{H}}, \theta, z\right) \\
\varepsilon_{\varphi \max } & =\operatorname{Max}\left\{\Delta \varphi\left(r_{\mathrm{H}}, \theta, z\right)\right\} \\
\overline{\varepsilon_{\varphi}} & =\frac{1}{N} \sqrt{\sum_{i=1}^{N} \sum_{j=1}^{N}\left[\Delta \varphi\left(r_{\mathrm{H}}, \theta, z\right)\right]^{2}}
\end{aligned}
$$

which $\varphi_{r e}\left(r_{\mathrm{H}}, \theta, z\right)$ is the reconstructed phase and $\varphi_{l}\left(r_{\mathrm{H}}, \theta, z\right)$ is the theoretical phase.

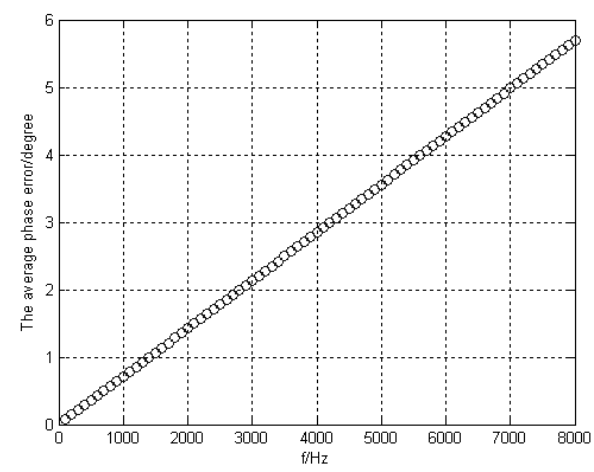

(a)The average phase error

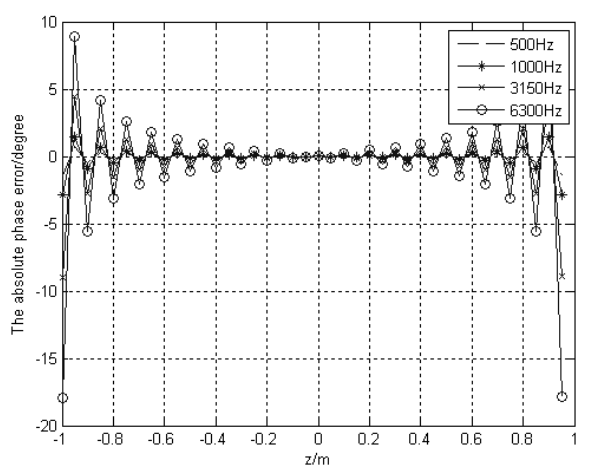

(b)The absolute phase error

Figure 1 Comparison of the phase errors of different frequency.

In fact, we hope that reconstruction work of the complex pressure with broadband frequency would be finished as much as we could, with the other invariable parameters. So 
we are always concerned about the relationship of the reconstructed frequency and the reconstructed phase error. Fig. 1 shows the distribution of the average reconstructed phase error $\overline{\mathcal{E}_{\varphi}}$ and the absolute error $\Delta \varphi$ which is calculated under different frequencies on the $\theta=0$ line.

From Fig. 1, when the other parameters are invariable, we can conclude that the average reconstructed phase error increases linearly along with the increase of the reconstructed frequency, moreover the amplitude of the absolute error and the maximal phase error increase gradually. Thus it is demonstrated that reconstructed results are good when this technique is applied to the low frequency sources, and become worse with the increase of the reconstructed frequency.

We assume that the reconstructed frequency is $1500 \mathrm{~Hz}$. Then we will discuss the effect of the holographic distance. Fig. 2 shows the distribution of the average reconstructed phase error $\overline{\mathcal{E}_{\varphi}}$ and the absolute error $\Delta \varphi$ which calculate on the $\theta=0$ line with the variety of the holographic distance $\mathrm{d}$ from $0.1 \lambda$ to $0.6 \lambda$. In Fig. 2, as the other parameters are invariable, the average reconstructed phase error decreases from $1.05^{\circ}$ to $0.85^{\circ}$ with the increase of holographic distance $\mathrm{d}$, that is, the average reconstructed phase error decreases linearly, and the amplitude of the absolute error increases gradually. In order to achieve the evanescent wave with detailed information about the source, the holographic distance must be small enough. Although the average error decreases with the increase of the holographic distance, the absolute error increases gradually, thus in practical measurement, the smaller holographic distance is preferable.

Provided that the aperture is invariable, then we study the effect of the holographic measuring points or measuring spacing on the reconstructed phase error. If $\delta$ is the measuring spacing, Fig.3 gives the variety of $\overline{\mathcal{E}_{\varphi}}$ and $\Delta \varphi$ on the $\theta=0$ line when $\delta$ increases from $0.05 \lambda$ to $0.4 \lambda$.

In Fig. 3, under the condition of invariable aperture, the increase of measuring spacing will bring about gradual decrease of the average reconstructed phase error. When the measuring spacing increase to twice, the amplitude of the absolute reconstructed phase error increases to twice or more, which is proportional to the increase of measuring spacing. Actually, although the decrease of measuring spacing will increase the resolution, it also brings more complexity to the measurement and restricts the reconstructed frequency. Therefore, in order to ensure the reconstructed precision, we should increase the measuring spacing as well as we can.

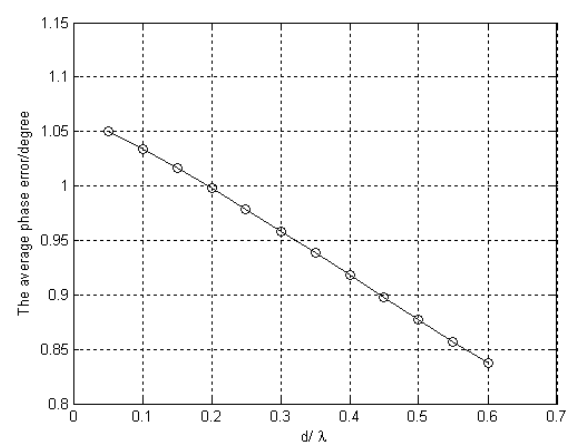

(a) The average phase error

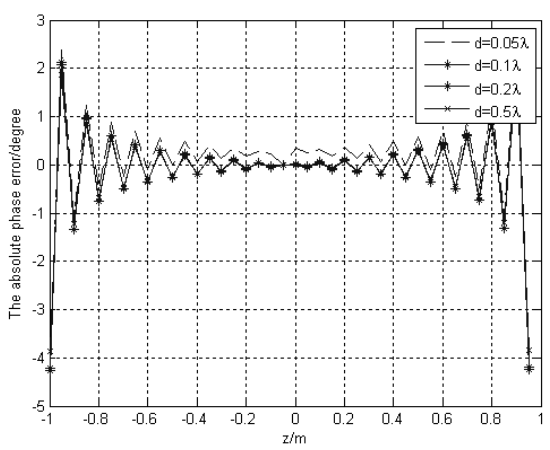

(b) The absolute phase error

Figure 2 Comparison of the phase errors of different $d$.

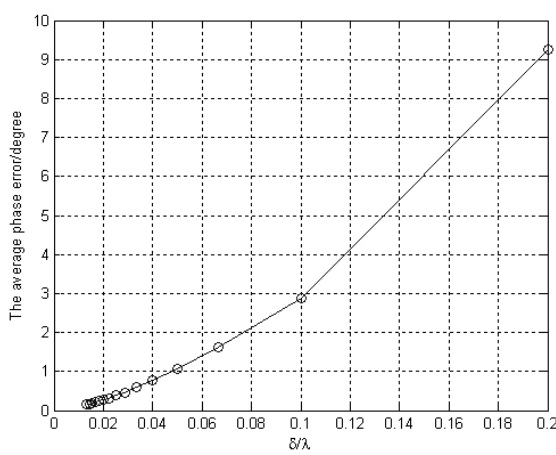

(a) The average phase error

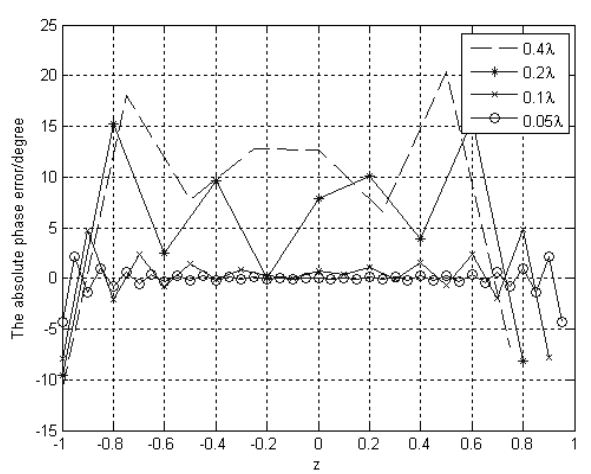

(b) The absolute phase error

Figure 3 Comparison of the phase errors of different measuring spacing 
Digital simulations produced for certain typical simple source demonstrate that: (1) With the increase of the reconstructed frequency, the reconstructed pressure phase error become worse. Compared with the high frequency sources, this technique gives better reconstructed result to the low frequency sources; (2) With the increase of the holographic distance, the average error of the reconstructed pressure phase decreases linearly and the amplitude of absolute phase error increases; (3) With the increase of the measuring spacing, the reconstructed phase error increase; (4) The system reconstructed parameters are restricted and influenced each other, thus they should be optimized in order to ensure the reconstructed veracity and provide the best measurement system.

\section{EXPERIMENTAL RESULTS}

Some experiments have been carried out in the anechoic pool to prove the correctness of parameter analysis. The measuring system is a vector hydrophone array $1.75 \mathrm{~m}$ long, which consists of 8 vector hydrophones spaced by $0.25 \mathrm{~m}$. During the experiment, the vector hydrophone array is put vertically in the water. It controlled by the computer does the axial rotation every 5 radian. Because it's difficult in completing the measurement of the whole sound field, we adjust the depth of the array by $0.08 \mathrm{~m}$ twice. The pressure and intensity on the cylindrical hologram surface with a radius of $0.52 \mathrm{~cm}$ is sampled at $-0.96 \mathrm{~m} \leq z \leq 0.96 \mathrm{~m}$. After the data collection, the sensitivity compensation and amplification amendment are made.

To begin with, the complex pressures phase needs to be calculated by intensity measurement. Then the reconstructed values will be compared with the theoretical values. Fig. 4 (a) and (b) are the reconstructed pressure phase and the absolute phase error respectively. The hologram surface is sampled at 72 -by-24 points in the circumferential directions and the axial, and the frequency is $3.15 \mathrm{kHz}$. This example demonstrates that the reconstructed pressure phase almost consist well with the theoretical values, and the absolute error appear fluctuating periodically. The maximal phase error in the middle area of holographic surface is below $5^{\circ}$. The experimental results are presented at a frequency of $4 \mathrm{kHz}$ in Fig. 5 with the other invariable parameters. Fig. 6 shows the experimental results when the sampling grids are 72-by-12 points in the circumferential directions and the axial, respectively, and the frequency is $3.15 \mathrm{kHz}$.

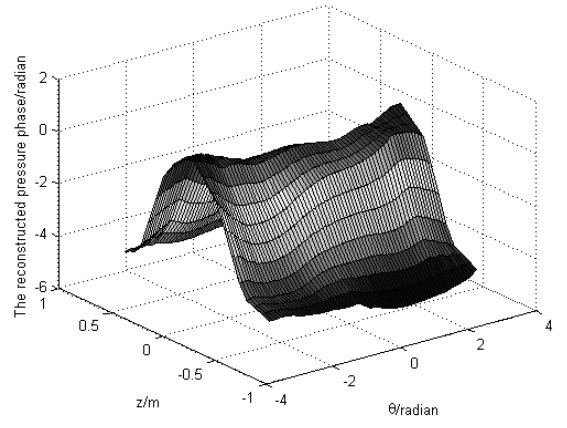

(a)The reconstructed pressure phase on holographic surface

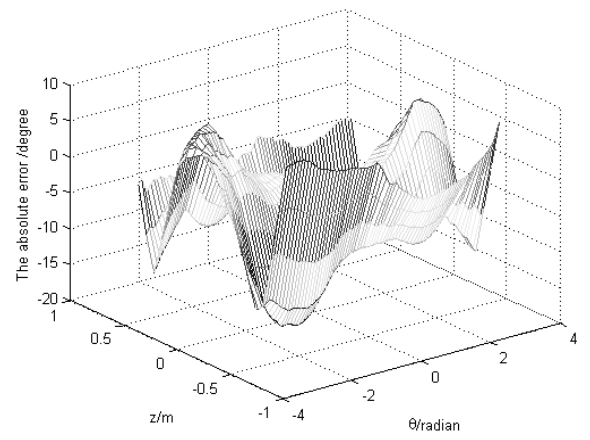

(b)The absolute error between theoretical and reconstructed phase

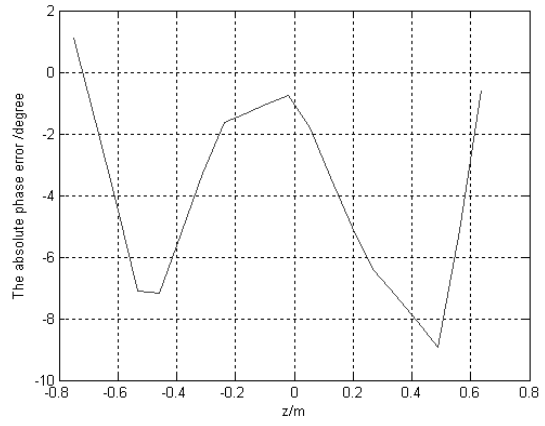

(c)The absolute phase error on cylinder $\theta=0$

Figure 4 Comparison of phase and distribution of phase error

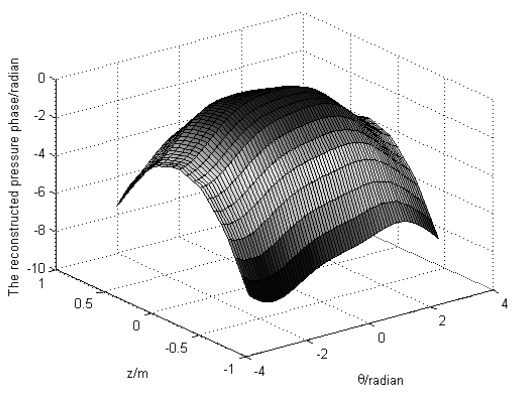

(a)The reconstructed pressure phase on holographic surface 


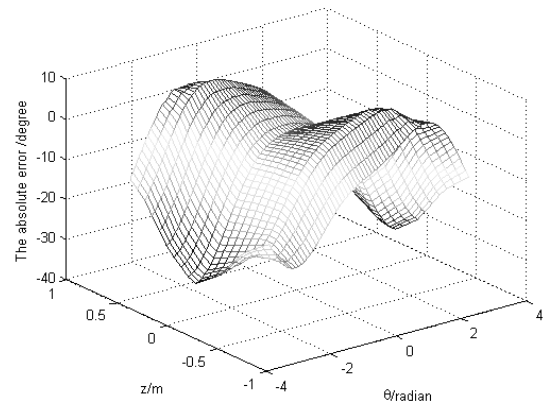

(b)The absolute error between theoretical and reconstructed phase

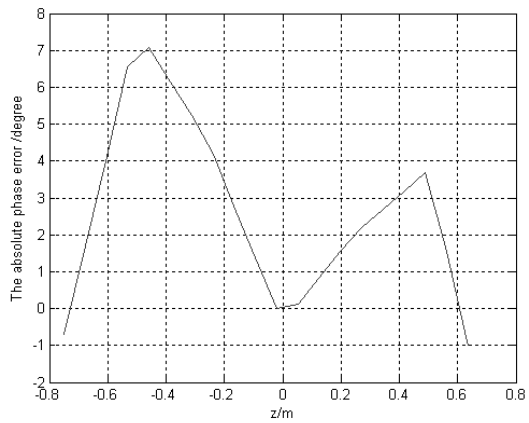

(c)The absolute phase error on cylinder $\theta=0$

Figure 5 Comparison of phase and distribution of phase error

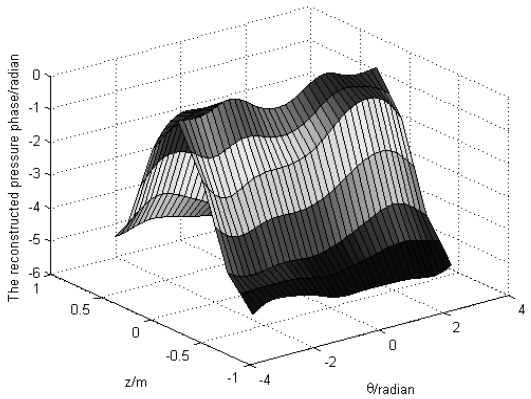

(a)The reconstructed pressure phase on holographic surface

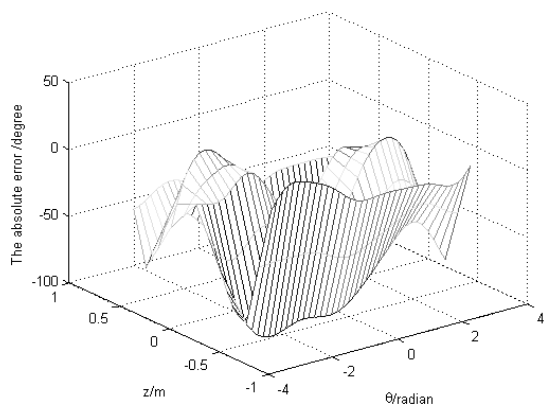

(b)The absolute error between theoretical and reconstructed phase

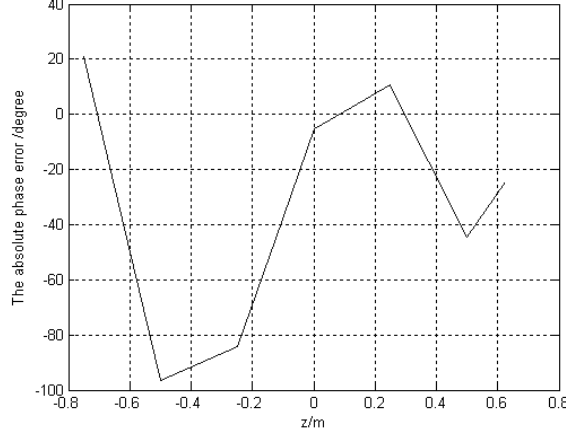

(c)The absolute phase error on cylinder $\theta=0$

Figure 6 Comparison of phase and distribution of phase error

Compared with the reconstructed results of above three figures, we can conclude that the reconstructed pressure phase error become worse with the increase of the reconstructed frequency and measuring spacing. The phase error is the most sensitive to the change of measuring spacing. The experimental results and simulation results have good consistency.

\section{CONCLUSION}

Using the BAHIM theory, the principle of the cylindrical interior BAHIM and implementation are presented and the numerical simulations are made as well. In addition, the influences of the change of parameters are discussed in detail. The results of experiment showed the merits of the BAHIM and the feasibility of the using of vector hydrophone array in the NAH measurement. And a wide range of applications for this method, which makes broadband work possible, are implied.

\section{REFERENCES}

[1] Maynard J D, Williams E G, Lee Y, "Nearfield acoustic holography (NAH): I. Theory of generalized holography and the development of NAH," J. Acoust. Soc. Am., vol.4, pp.1395-1413, 1985

[2] Williams E G, Dardy H D, "Generalized nearfield acoustical holography for cylindrical geometry: Theory and experiment," J. Acoust. Soc. Am., vol.2, pp. 389-407, 1987

[3] J. Hald. "Patch near-field acoustic holography using a new statistically optimal method". Proceedings of INTER-NOISE 2003, 2003, pp. 2203-2210

[4] Steiner R, Hald J. "Near-field acoustic holography without the errors and limitations caused by the use of spatial DFT". International Journal of Acoustics and Vibration, vol.6, pp. 83-89, 2001

[5] Loyau T., Pascal J. C., Gaillard P., "Broadband acoustic holography reconstruction from acoustic intensity measurements. I: Principle of the method.," J Acoust. Sco. Am.,vol. 5,pp.1744-1750, 1985

[6] Williams E. G., Houston B H, Gucaro J A, "Broadband nearfild acoustical holography for vibrating cylinder," J Acoust. Sco. Am., vol. 4, pp.674-679, 1989

[7] Hu Bo, Yang De Sen. "Research on implementation and application of underwater cylindrical broadband acoustic holography". Journal of Harbin Engineering University, vol. 29,pp. 382-389, 2008 